übereinstimmend erachten. In Rectascension geben die Aitken'schen Messungen der Nova eine entschieden zu grosse negative Bewegung, obwohl der Sinn dieser Bewegung ganz bestimmt ausgesprochen ist. Hier mussen also systematische Auffassungsfehler der Nova-Bilder zu einer der beiden Zeiten falschend gewirkt haben, da die recht symmetrische Lage der 6 Sterne um die Nova systematische Messungsfehler ausschliesst. Man wird wohl annehmen dürfen, dass eine Deformation des hellen Bildes der Nova zur Zeit der ersten Messungsreihe in dem lichtstarken Fernrohr einen Auffassungsfehler - und zwar müsste derselbe die Rectascensionen zu gross haben messen lassen - erzeugte, zumal da der einzige Stern Aitken's, dessen Position auch in AG. Bonn vorkommt, $p=$ Bonn 2948, für den Ort der Nova

Berlin-Friedenau, 1902 Jan. 12. ergiebt: $3^{\mathrm{h}} 24^{\mathrm{m}} 28^{\mathrm{s}} .29+43^{\circ} 33^{\prime} 53^{\prime \prime} 7$ Epoche 1901.18, also gegen Bergstrand's Messungen eine gleichfalls zu grosse Rectascension finden lässt.

Mehr wie eine Bestätigung der Bergstrand'schen Eigenbewegung dem Sinne nach wird man aber von Aitken's Refractormessungen auch nicht erwarten dürfen, als eine solche aber dürfen sie auch wohl betrachtet werden.

Es mag noch darauf hingewiesen werden, dass eine jährliche seitliche E. B. der Nova von $I^{\prime \prime}$ bei einer Parallaxe von 0.०Ix auf eine Geschwindigkeit von $430 \mathrm{~km}$ in der Secunde führen würde, welche, da die Geschwindigkeit im Visionsradius nach Campbell nur $+6 \mathrm{~km}$ beträgt, auch der Gesammtbewegung der Nova entspricht und damit eine der grössten überhaupt bekannten ist.

\section{F. Ristenpart.}

\title{
Bemerkungen betreffend den Stern BD. $+1^{\circ} 579$.
}

Als Vergleichstern für den Planeten I $9 \circ \mathrm{I} \mathrm{HJ}$ benutzte ich 1901 Nov. 16 den Stern BD. +19578 , dessen aus den AG.- Catalogen auf 1855.0 reducirter Ort so lautet:

$$
9: 23^{\mathrm{h}} 12^{\mathrm{m}} 15^{5: 8}+1^{\circ} 8: 7 \text {. }
$$

Ich vermisste bei dieser Gelegenheit den Stern $9 \cdot 3$ BD. $+1: 579$, welcher nach der BD. $+2.0-0.2$ südlich auf +19578 folgen soll, gewahrte aber einen in BD. fehlenden Stern $9: 3$, dessen Ort ich Nov, 17 durch mikrometrischen Anschluss an $1 / 2$ (Nicol. $695+$ Alb. 936) so bestimmte :

$$
\begin{aligned}
& 190 \mathrm{r} .09^{\mathrm{m}} \cdot 33^{\mathrm{h}} \mathrm{r}^{\mathrm{m}} 3^{0.6} \cdot 6+\mathrm{r}^{\circ} 22^{\prime} \quad 8 \%^{\circ} \circ \\
& \text { also } \quad 1855.0 \quad 3128.4+\text { I II.9. }
\end{aligned}
$$

Herr Prof. Kùstner hat die Güte gehabt, die Originalbeobachtungen der BD. nachzusehen, und theilt mir darüber folgendes mit:

- Die Sterne $+1{ }_{578}^{\circ}$ und $+1{ }_{579}^{\circ}$ sind in $\mathrm{BD}$, wie folgt zusammen beobachtet:

$$
\text { Düsseldorf, 1901 Nov. } 25 .
$$

$$
\begin{array}{ccl}
\text { Kr. } 283 & 1853 & \text { Dec. } 3 \\
& 9^{\mathrm{m}} & 3^{\mathrm{h}} 12^{\mathrm{m}} 13^{\mathrm{s}} 6+1^{\circ} 9: 4 \\
9 & 15.6 & 8.9,
\end{array}
$$

alles deutlich und richtig reducirt.

In dieser Zone ist auch beobachtet:

$$
9^{\mathrm{m}} \cdot 53^{\mathrm{h}} 12^{\mathrm{m}} 4^{\mathrm{s}} \cdot 6+1^{\circ} 12 ! 3 \text {, }
$$

welcher vermuthlich mit dem von Ihnen bobachteten Stern $9 \cdot 3$ identisch ist; als einmal beobachtet ist dieser nicht in BD. aufgenommen.

$$
\begin{aligned}
& \text { Sch. } 276 \text { I } 853 \text { Dec. } 2 \\
& 9^{\mathrm{m}} \quad 3^{\mathrm{h}} 12^{\mathrm{m}} 16^{\mathrm{s}} .0+1^{\circ} 7^{\circ} 6 \text { sequitur } 9^{\mathrm{m}} \cdot 5 \\
& \begin{array}{lll}
9.5 & (\mathrm{I} 8) \quad 6.9
\end{array}
\end{aligned}
$$

$+1: 579$ ist hier nachbeobachtet, die RA. offenbar ergänzt nach Kr.'s Beobachtung; die Identificirung erschien zweifelsfrei wegen der Bemerkung bei dem Bessel'schen Stern $+1: 578$ sequitur $9{ }^{m} \cdot 5 \ll$. - Demgemäss ist dann der Ort von $+1: 579$ in $\mathrm{BD}$. angesetzt worden; die AR. 2:0 grösser als $+1{ }^{\circ} 578$, wie sie. $\mathrm{Kr}$. beobachtet hatte.*

\section{W. Luther.}

\section{Literarische Anzeigen.}

Julius Baurschinger. Tafeln zur theoretischen Astronomie. Leipzig 1901.

Die vorliegende, aus der astronomischen Praxis hervorgegangene Tafelsammlung wird sicher sehr bald ein unentbehrliches Hülfsmittel für den rechnenden Astronomen werden. Wegen des reichhaltigen Inhalts sei auf die dieser Nummer beiliegende Anktundigung der Firma W. Engelmann in Leipzig verwiesen.

J. H. Pernter. Meteorologische Optik. I. Abschnitt. Wien und Leipzig I 902.

Zufolge der Bedeutung der meteorologischen Optik für die beobachtende Astronomie wird ein kurzer Hinweis auf die vorliegende wichtige Publication am Platze sein. Der zunächst allein publicirte I. Abschnitt behandelt die scheinbare Gestalt des Himmelsgewölbes, die scheinbare Vergrösserung von Sonne und Mond am Horizonte und andere hiermit zusammenhängende Erscheinungen.

Inhalt zu Nr. 3763. A. Scheller. Untersuchung der Bahn des Cometen 1845 II. 309. - W. Stratonow. Beobachtung der Sonnenfinsterniss Igol Nov. I1. 317. - A. A. Nijland. Beobachtungen des Cometen I901 I. 319. - F. G. Hagen, S. J. Ueber Ceraski's Begleiter der Nova Persei. $32 \mathrm{I}$ - F. Ristenpart. Zur Frage der Eigenbewegung der Nova Persei. $32 \mathrm{I}$. - W. Luther. Bemerkungen betreffend den Stern BD. +19579. 323. - Literarische Anzeigen. 323. 